\title{
ON MULTIPLE SOLUTIONS \\ OF THE EXTERIOR NEUMANN PROBLEM INVOLVING CRITICAL SOBOLEV EXPONENT
}

\author{
Jan Chabrowski - Michel Willem
}

Dedicated to the memory of Professor Olga A. Ladyzhenskaya

AbStract. In this paper we consider the exterior Neumann problem involving a critical Sobolev exponent. We establish the existence of two solutions having a prescribed limit at infinity.

\section{Introduction}

Let $\Omega \subset \mathbb{R}^{N}$ be a bounded domain with a smooth boundary $\partial \Omega$. We set $\Omega^{c}=\mathbb{R}^{N}-\bar{\Omega}$. We consider the Neumann problem on the exterior domain $\Omega^{c}$

$\left(1_{\mu}\right) \quad \begin{cases}-\Delta u=Q(x) u^{2^{*}-1} & \text { in } \Omega^{c}, \\ \frac{\partial u}{\partial \nu}=0 & \text { on } \partial \Omega, u>0 \text { on } \bar{\Omega}^{c}, \\ \lim _{|x| \rightarrow \infty} u(x)=\mu>0, & \end{cases}$

where $2^{*}=2 N /(N-2), N \geq 3$, is a critical Sobolev exponent and $\mu>0$ is a given parameter. We assume that the coefficient $Q$ is locally Hölder continuous on $\Omega^{c}, Q(x)>0$ on $\Omega^{c}$ and

$\left(Q_{1}\right)$

$$
Q(x) \leq C|x|^{r}
$$

2000 Mathematics Subject Classification. 35B33, 35J65, 35Q55.

Key words and phrases. Neumann problem, critical Sobolev exponent, multiple solutions. Supported by NATO grant PST.CLG.978736. 
for some constant $C>0$ and $r<-2$ and large $|x|$. More specific conditions on $r$ will be given later. The novelty here is that we consider the exterior Neumann problem with a critical Sobolev exponent and with a prescribed limit at infinity. A similar problem in the case of the Dirichlet problem has been considered in the paper [6]. In the present paper we show the existence of two solutions. The first one is obtained through the method of sub and super-solutions. This solution will be used to translate the variational functional for $\left(1_{\mu}\right)$ and then apply the mountain-pass principle to get a second solution.

In this paper we use standard notations. By $D^{1,2}\left(\Omega^{c}\right)$ we denote the Sobolev space defined by

$$
D^{1,2}\left(\Omega^{c}\right)=\left\{u: u \in L^{2^{*}}\left(\Omega^{c}\right),|\nabla u| \in L^{2}\left(\Omega^{c}\right)\right\},
$$

equipped with the norm

$$
\|u\|_{L^{2^{*}}\left(\Omega^{c}\right)}+\||\nabla u|\|_{L^{2}\left(\Omega^{c}\right)} .
$$

This norm is equivalent to the norm $\||\nabla u|\|_{L^{2}\left(\Omega^{c}\right)}$ (see $[9]$ ). The space $D^{1,2}\left(\Omega^{c}\right)$ is a natural space for the translated variational functional corresponding to problem $\left(1_{\mu}\right)$. Let

$$
S\left(\Omega^{c}\right)=\inf _{\substack{\phi \in D^{1,2}\left(\Omega^{c}\right) \\ \phi \neq 0}} \frac{\int_{\Omega^{c}}|\nabla \phi|^{2} d x}{\left(\int_{\Omega^{c}}|\phi|^{2^{*}} d x\right)^{(N-2) / N}} .
$$

It is known [11] that if the mean curvature of $\partial \Omega$, when seen from inside of $\Omega$, is negative somewhere, then

$$
S\left(\Omega^{c}\right)<\frac{S}{2^{2 / N}},
$$

where $S$ is the usual best Sobolev constant, i.e.

$$
S=\inf _{\substack{\phi \in D^{1,2}\left(\mathbb{R}^{N}\right) \\ \phi \neq 0}} \frac{\int_{\mathbb{R}^{N}}|\nabla \phi|^{2} d x}{\left(\int_{\mathbb{R}^{N}}|\phi|^{2^{*}} d x\right)^{(N-2) / N}} .
$$

Here $D^{1,2}\left(\mathbb{R}^{N}\right)$ is a Sobolev space defined by

$$
D^{1,2}\left(\mathbb{R}^{N}\right)=\left\{u: u \in L^{2^{*}}\left(\mathbb{R}^{N}\right),|\nabla u| \in L^{2}\left(\mathbb{R}^{N}\right)\right\} .
$$

Thus if (s) holds, then $S\left(\Omega^{c}\right)$ is achieved. Moreover, if $\Omega=B(0, R)$, or $\Omega$ is close to a ball, then $S\left(\Omega^{c}\right)=S / 2^{2 / N}$ (see [11]).

In a given Banach space $X$ we denote a strong convergence by " $\rightarrow$ " and weak convergence by "—". We recall that a $C^{1}$-functional $\Phi: X \rightarrow \mathbb{R}$ on a Banach space $X$ satisfies the Palais-Smale condition at level $c\left((\mathrm{PS})_{c}\right.$ condition for short), if each sequence $\left\{x_{m}\right\}$ such that

$(*) \Phi\left(x_{m}\right) \rightarrow c$, and

$(* *) \Phi^{\prime}\left(x_{m}\right) \rightarrow 0$ in $X^{*}$ 
is relatively compact in $X$. Finally, any sequnce satisfying $(*)$ and $(* *)$ is called a Palais-Smale sequence at level $c$ (a (PS) ${ }_{c}$ sequence for short).

The norms in the Lebesgue spaces $L^{q}\left(\Omega^{c}\right)$ will be denoted by $\|\cdot\|_{q}$.

\section{Minimal solution}

In this section we establish the existence of a solution of $\left(1_{\mu}\right)$ through the method of sub and super-solutions.

To construct a supersolution we need the solution of the problem

$$
\begin{cases}-\Delta w=Q(x) & \text { in } \Omega^{c}, \\ \frac{\partial w}{\partial \nu}=0 & \text { on } \partial \Omega, \\ \lim _{|x| \rightarrow \infty} w(x)=0 . & \end{cases}
$$

Lemma 2.1. Problem (2.1) has a solution satisfying

$$
0<w(x) \leq \begin{cases}C|x|^{2-N} & \text { if } r<-N, \\ C|x|^{2-N} \log |x| & \text { if } r=-N, \\ C|x|^{2+r} & \text { if }-N<r<-2,\end{cases}
$$

for large $|x|$ and some constant $C>0$.

Proof. Let $m_{\circ} \in \mathbb{N}$ be such that $\Omega \subset B\left(0, m_{\circ}\right)$. For each $m>m_{\circ}$ we consider the problem

$$
\begin{cases}-\Delta u=Q(x) & \text { in } \Omega^{c} \cap B(0, m), \\ \frac{\partial u}{\partial \nu}=0 & \text { on } \partial \Omega, \\ u=0 & \text { on } \partial B(0, m) .\end{cases}
$$

For each $m \geq m_{\circ}$ problem $\left(1_{m}\right)$ has a solution $u_{m}$. We extend $u_{m}$ by 0 outside $B(0, m)$. By the maximum principle the sequence $\left\{u_{m}\right\}$ is increasing and uniformly bounded. By the Schauder estimates (see [8]) we may assume that $u_{m} \rightarrow w$ in $C^{2}\left(\Omega^{c} \cap B(0, R)\right)$ and $C^{1}\left(\bar{\Omega}^{c} \cap B(0, R)\right)$ for each $R>0$ large. Obviously $w>0$ on $\Omega^{c}$ and $w$ satisfies the equation and the boundary condition in (2.1). To show that $w$ satisfies (2.2), we introduce a function $z(x)$ which is a solution of the exterior Dirichlet problem

$$
\begin{cases}-\Delta z=Q(x) \quad \text { in } \Omega^{c}, \\ z=0 & \text { on } \partial \Omega, \\ \lim _{|x| \rightarrow \infty} z(x)=0 . & \end{cases}
$$

The function $z$ is positive on $\Omega^{c}$ and satisfies (2.2) (see [6]). Since $\left\{u_{m}\right\}$ are uniformly bounded on $\Omega^{c}$, there exists a constant $C>1$ such that $u_{m}(x) \leq C z(x)$ for $x \in \partial B\left(0, m_{\circ}\right)$ and $m>m_{\circ}$. Moreover, $u_{m}(x)=0$ for $x \in \partial B(0, m)$ and

$$
-\Delta\left(u_{m}-C z\right)=Q(x)-C Q(x)<0 \quad \text { on } B(0, m)-B\left(0, m_{\circ}\right) .
$$


Hence by the maximum principle $u_{m} \leq C z$ on $\mathbb{R}^{N}-B\left(0, m_{\circ}\right)$ for every $m>m_{\circ}$. Letting $m \rightarrow \infty$ we get $w(x) \leq C z(x)$ and the result follows.

LEMma A. Suppose that

$(\mathrm{H}) Q: \bar{\Omega}^{c} \rightarrow \mathbb{R}$ is locally Hölder continuous, $Q(x)>0$ and $Q(x) \leq c|x|^{r}$ on $\bar{\Omega}^{c}$, where $r<-(N+2) / 2$ and $c>0$.

Then the problems (2.1) and

$$
\begin{cases}-\Delta w=Q(x), w(x)>0 & \text { in } \Omega^{c}, \\ \frac{\partial w}{\partial \nu}=0 & \text { on } \left.\partial \Omega, w \in D^{1,2} \Omega^{c}\right),\end{cases}
$$

are equivalent. Moreover, the solution of (2.1) (or (2.1')) exists and is unique.

Proof. Since

$$
Q \in L^{2 N /(N+2)}\left(\Omega^{c}\right) \cong\left(L^{2^{*}}\left(\Omega^{c}\right)\right)^{\prime},
$$

it follows from the Riesz-Fréchet representation theorem that (2.1') has a unique solution $w_{\circ}$ in $D^{1,2}\left(\Omega^{c}\right)$. On the other hand the problem

$$
\begin{cases}-\Delta u=0 & \text { in } \Omega^{c} \\ \frac{\partial u}{\partial \nu}=0 & \text { on } \partial \Omega \\ \lim _{|x| \rightarrow \infty} u(x)=0 & \end{cases}
$$

has a unique solution $u \equiv 0$ (see [6]). Hence by Lemma 2.1, problem (2.1) has a unique solution, say $w_{1}$. Since by Lemma $2.1 w_{1} \in D^{1,2}\left(\Omega^{c}\right), w_{1} \equiv w_{\circ}$.

Lemma B. Suppose that the assumption $(\mathrm{H})$ holds. Then problems

$$
\begin{cases}-\Delta u=Q(x), \quad u(x)>\mu>0 & \text { in } \Omega^{c} \\ \frac{\partial u}{\partial \nu}=0 & \text { on } \partial \Omega^{c} \\ \lim _{|x| \rightarrow \infty} u(x)=\mu & \end{cases}
$$

and

$$
\begin{cases}-\Delta u=Q(x), \quad \mu>0 & \text { on } \Omega^{c}, \\ \frac{\partial u}{\partial \nu}=0 & \text { on } \partial \Omega,(u-\mu) \in D^{1,2}\left(\Omega^{c}\right)\end{cases}
$$

are equivalent and have a unique solutions.

Proof. Define $u=w+\mu$ and apply Lemma A.

To proceed further we introduce the definition of a subsolution and supersolution of $\left(1_{\mu}\right)$.

We say that a function $\phi>0$ on $\Omega^{c}$ is a supersolution of $\left(1_{\mu}\right)$ if $\phi \in$ $C^{2}\left(\Omega^{c}\right) \cap C^{1}\left(\bar{\Omega}^{c}\right),-\Delta \phi \geq Q \phi^{p}$, where $p=2^{*}-1$, on $\Omega^{c}, \partial \phi / \partial \nu=0$ on $\partial \Omega$ and $\lim _{|x| \rightarrow \infty} \phi(x) \geq \mu$.

The definition of a subsolution $\psi>0$ is obtained by reversing the inequalities in the above definition. 
If problem $\left(1_{\mu}\right)$ has a subsolution $\psi$ and a supersolution $\phi$ such that $0<\psi<$ $\phi$ on $\Omega^{c}$, then problem $\left(1_{\mu}\right)$ has a minimal solution $\underline{u}$ and a maximal solution $\bar{u}$ such that $\psi \leq \underline{u} \leq \bar{u} \leq \phi$ on $\Omega^{c}$. This can be established by employing a standard monotone iteration technique. First we observe that if $w$ is the solution of (2.1) then the function $w_{\mu}=\mu+w$ is the unique solution of the following problem

$$
\begin{cases}-\Delta u=Q(x) & \text { in } \Omega^{c}, \\ \frac{\partial u}{\partial \nu}=0 & \text { on } \partial \Omega, \\ \lim _{|x| \rightarrow \infty} u(x)=\mu . & \end{cases}
$$

Let $u_{0}=\phi$ and for every $j \geq 1$ we define $u_{j}$ as a solution of the problem

$$
\begin{cases}-\Delta u_{j}=Q(x) u_{j-1}^{p} & \text { in } \Omega^{c}, \\ \frac{\partial u_{j}}{\partial \nu}=0 & \text { on } \partial \Omega, \\ \lim _{|x| \rightarrow \infty} u_{j}(x)=\mu . & \end{cases}
$$

By the maximum principle we have

$$
u_{j} \leq u_{j-1} \leq \ldots \leq u_{1} \leq u_{0} \text { on } \Omega^{c} .
$$

Similarly, we set $v_{0}=\psi$. Let $v_{j}$ for $j \geq 1$ be a solution of the problem

$$
\begin{cases}-\Delta v_{j}=Q(x) v_{j-1}^{p} & \text { in } \Omega^{c}, \\ \frac{\partial v_{j}}{\partial \nu}=0 & \text { on } \partial \Omega, \\ \lim _{|x| \rightarrow \infty} v_{j}(x)=\mu . & \end{cases}
$$

By the maximum principle we have

$$
\psi=v_{0} \leq v_{1} \leq \ldots \leq v_{j} \text { on } \Omega^{c} .
$$

Also, we have $v_{j} \leq u_{j}$ on $\Omega^{c}$. Taking the limits of the sequences $\left\{v_{j}\right\}$ and $\left\{u_{j}\right\}$ we obtain a minimal solution $\underline{u}$ and a maximal solution $\bar{u}$.

To apply the above method, let $w_{1}$ be a solution of (2.3) with $\mu=1$. Then we set $\phi_{\mu}=\mu w_{1}$ and $\psi_{\mu}=\mu$. It is clear that $\psi_{\mu}<\phi_{\mu}$ on $\Omega^{c}$ and $\lim _{|x| \rightarrow \infty} \phi_{\mu}(x)=$ $\mu$. We now observe that

$$
-\Delta \phi_{\mu}-Q(x) \phi_{\mu}^{2^{*}-1}=\mu Q(x)-Q(x)\left(\mu w_{1}\right)^{2^{*}-1}=Q(x) \mu\left(1-\mu^{2^{*}-2} w_{1}^{2^{*}-1}\right) \geq 0
$$

on $\Omega^{c}$ for $\mu$ small, say $0<\mu \leq \mu_{\circ}$. Obviously, $\psi$ is a subsolution for $\left(1_{\mu}\right)$. By the method of sub and supersolutions problem $\left(1_{\mu}\right)$ has a minimal solution $u_{\mu}$ satisfying $\mu \leq u_{\mu} \leq \phi_{\mu}$ for $0<\mu \leq \mu_{\circ}$.

We let

$$
\bar{\mu}=\sup \left\{\mu>0: \text { problem }\left(1_{\mu}\right) \text { has a solution }\right\} \text {. }
$$


Proposition 2.2. Suppose that the assumption $(\mathrm{H})$ holds. Problem $\left(1_{\mu}\right)$ has a solution for every $0<\mu<\bar{\mu}$. Moreover, $0<\bar{\mu}<\infty$ and there are no solutions for $\mu>\bar{\mu}$.

Proof. Let $\mu \in(0, \bar{\mu})$. Then there exists $\widetilde{\mu} \in(\mu, \bar{\mu})$ such that problem $\left(1_{\tilde{\mu}}\right)$ has a solution $u_{\tilde{\mu}}$. This solution $u_{\tilde{\mu}}$ is a supersolution of $\left(1_{\mu}\right)$ and $v=\mu$ is a subsolution of $\left(1_{\mu}\right)$. Hence problem $\left(1_{\mu}\right)$ has a minimal solution $u_{\mu}$ such that $\mu \leq u_{\mu} \leq u_{\tilde{\mu}}$. Arguing by contradiction, assume that $\bar{\mu}=\infty$. Then for every $\mu>0$ there exists a minimal solution $u_{\mu}$. Letting $v=u_{\mu}-\mu$, we see that

$$
-\Delta v=-\Delta u_{\mu}^{2^{*}-1} \geq Q(x) \mu^{2^{*}-2}\left(u_{\mu}-\mu\right)=Q(x) \mu^{2^{*}-2} v
$$

and $v>0$ on $\Omega^{c}$. By Lemma B $v \in D^{1,2}\left(\Omega^{c}\right)$. Hence the first eigenvalue for $-\Delta-Q(x) \mu^{2^{*}-2}$ is nonnegative. On the other hand for large $\mu$, the first eigenvalue must be negative and we have reached a contradiction.

\section{Properties of minimal solutions}

From Lemma B we deduce the following estimate for $u_{\mu}-\mu$.

Lemma 3.1. Suppose that the assumption $(\mathrm{H})$ holds. Let $u_{\mu}$ be the minimal solution of $\left(1_{\mu}\right)$ from Proposition 2.2. Then

$$
0<u_{\mu}-\mu \leq \begin{cases}C|x|^{2-N} & \text { if } r<-N, \\ C|x|^{2-N} \log |x| & \text { if } r=-N, \\ C|x|^{2+r} & \text { if }-N<r<-2,\end{cases}
$$

for some constant $C>0$ and large $|x|$.

Lemma 3.2. Suppose $(\mathrm{H})$ holds. Further, we assume that $u$ is a bounded positive solution of $\left(1_{\mu}\right)$ such that $u-\mu \in D^{1,2}\left(\Omega^{c}\right)$. Then the variational problem

$$
\sigma_{\mu}=\inf \left\{\int_{\Omega^{c}}|\nabla w|^{2} d x: w \in D^{1,2}\left(\Omega^{c}\right), p \int_{\Omega^{c}} Q(x) u^{p-1} w^{2} d x=1\right\},
$$

where $p=2^{*}-1$, has a minimizer $\psi_{\mu}$ satisfying

$$
\begin{cases}-\Delta \psi_{\mu}=p \sigma_{\mu} Q(x) u^{p-1} \psi_{\mu} & \text { in } \Omega^{c} \\ \frac{\partial \psi_{\mu}}{\partial \nu}=0 & \text { on } \partial \Omega .\end{cases}
$$

If there exists a bounded positive solution $\bar{u}$ of $\left(1_{\bar{\mu}}\right)$ with $\bar{\mu}>\mu$ and such that $\bar{u}>u$ on $\Omega^{c}$ and $\bar{u}-\bar{\mu} \in D^{1,2}\left(\Omega^{c}\right)$, then $\sigma_{\mu}>1$.

Proof. The first part of the lemma follows from the fact that the functional $w \in D^{1,2}\left(\Omega^{c}\right) \rightarrow \int_{\Omega^{c}} Q(x) u^{p-1} w^{2} d x$ is weakly sequentially compact. Here we need the assumption $\left(Q_{1}\right)$. We only give the proof of the second part. We set $v=u-\mu$ and $\bar{v}=\bar{u}-\bar{\mu}$. Then

$$
-\Delta(\bar{v}-v)=Q(x)(\bar{v}+\bar{\mu})^{p}-Q(x)(v+\mu)^{p}=Q(x)\left(\bar{u}^{p}-u^{p}\right) \geq 0,
$$


$\partial(\bar{v}-v) / \partial \nu=0$ on $\partial \Omega$ and $\bar{v}-v \rightarrow 0$ as $|x| \rightarrow \infty$. Therefore by the maximum principle $\bar{v}>v$ on $\Omega^{c}$. We now observe that

$$
\begin{cases}-\Delta(\bar{v}-v)=Q(x)\left(\bar{u}^{p}-u^{p}\right) \geq p Q(x) u^{p-1}(\bar{v}-v+(\bar{\mu}-\mu)) & \text { in } \Omega^{c} \\ \frac{\partial(\bar{v}-v)}{\partial \nu}=0, \quad \bar{v}-v \in D^{1,2}\left(\Omega^{c}\right) & \text { on } \partial \Omega .\end{cases}
$$

Let $w=\bar{u}-u$. Testing (3.2) with $\psi_{\mu}$ we get

$$
\int_{\Omega^{c}} \nabla \psi_{\mu} \nabla w d x \geq p \int_{\Omega^{c}} Q(x) u^{p-1}(w+(\bar{\mu}-\mu)) \psi_{\mu} d x .
$$

On the other hand since $\psi_{\mu}$ is a solution of (3.1), we get

$$
\int_{\Omega^{c}} \nabla \psi_{\mu} \nabla w d x=p \sigma_{\mu} \int_{\Omega^{c}} Q(x) u^{p-1} \psi_{\mu} w d x .
$$

Then (3.2) and (3.3) imply that

$$
p \sigma_{\mu} \int_{\Omega^{c}} Q(x) u^{p-1} w \psi_{\mu} d x>p \int_{\Omega^{c}} Q(x) u^{p-1} w \psi_{\mu} d x .
$$

This shows that $\sigma_{\mu}>1$.

Lemma 3.2 can be applied to a family of minimal solutions $\left\{u_{\mu}\right\}, 0<\mu<\bar{\mu}$, since by Lemma B $u_{\mu}-\mu \in D^{1,2}\left(\Omega^{c}\right)$. Taking in Lemma $3.2 u=u_{\mu}$ for $0<\mu<\bar{\mu}$, we see that the corresponding $\sigma_{\mu}>1$. However, Lemma 3.2 cannot be applied to $u_{\bar{\mu}}$. Later we shall show that $\sigma_{\bar{\mu}}=1$.

Lemma 3.3. Suppose $(\mathrm{H})$ holds. Then there exists a constant $C>0$ independent of $\mu$ such that

$$
\left\|\nabla\left(u_{\mu}-\mu\right)\right\|_{2} \leq C
$$

for every $0<\mu<\bar{\mu}$.

Proof. Let $v_{\mu}=u_{\mu}-\mu$. Then by Lemma B we have

$$
\int_{\Omega^{c}}\left|\nabla v_{\mu}\right|^{2} d x=\int_{\Omega^{c}} Q(x)\left(v_{\mu}+\mu\right)^{p} v_{\mu} d x .
$$

Applying Lemma 3.2 we get

$$
\int_{\Omega^{c}}\left|\nabla v_{\mu}\right|^{2} d x \geq p \sigma_{\mu} \int_{\Omega^{c}} Q(x)\left(v_{\mu}+\mu\right)^{p-1} v_{\mu}^{2} d x d x .
$$

Combining these two relations we get

$$
\begin{aligned}
p \int_{\Omega^{c}} Q(x) v_{\mu}^{p+1} d x \leq & p \sigma_{\mu} \int_{\Omega^{c}} Q(x)\left(v_{\mu}+\mu\right)^{p-1} v_{\mu}^{2} d x d x \\
\leq & \int_{\Omega^{c}} Q(x)\left(v_{\mu}+\mu\right)^{p} v_{\mu} d x \\
= & \int_{\Omega^{c}} Q(x)\left(v_{\mu}+\mu\right)^{p-1} v_{\mu}^{2} d x d x \\
& +\int_{\Omega^{c}} Q(x)\left(v_{\mu}+\mu\right)^{p-1} \mu v_{\mu} d x .
\end{aligned}
$$


Hence by the Hölder and Young inequalities, we have for every $\varepsilon>0$

$$
\begin{aligned}
&(p-1) \int_{\Omega^{c}} Q(x)\left(u_{\mu}+\mu\right)^{p-1} v_{\mu}^{2} d x \leq \int_{\Omega^{c}} Q(x)\left(v_{\mu}+\mu\right)^{p-1} \mu v_{\mu} d x \\
& \leq C\left(\int_{\Omega^{c}} Q(x) v_{\mu}^{p} d x+\int_{\Omega^{c}} Q(x) v_{\mu} d x\right) \\
& \leq C\left(\int_{\Omega^{c}} Q(x) d x\right)^{1 /(p+1)}\left(\int_{\Omega^{c}} Q(x) v_{\mu}^{p+1} d x\right)^{p /(p+1)} \\
&+C\left(\int_{\Omega^{c}} Q(x) d x\right)^{p /(p+1)}\left(\int_{\Omega^{c}} Q(x) v_{\mu}^{p+1} d x\right)^{1 /(p+1)} \\
& \leq \varepsilon \int_{\Omega^{c}} Q(x) v_{\mu}^{p+1} d x+C_{\varepsilon} \int_{\Omega^{c}} Q(x) d x .
\end{aligned}
$$

Taking $\varepsilon>0$ sufficiently, small we derive from this inequality and (3.5) that

$$
\int_{\Omega^{c}} Q(x) v_{\mu}^{p+1} d x \leq C \int_{\Omega^{c}} Q(x) d x .
$$

The desired result follows from (3.4) and (3.6) with the aid of the Hölder inequality.

We show below that problem $\left(1_{\mu}\right)$ is also solvable for $\mu=\bar{\mu}$.

Proposition 3.4. Suppose $(\mathrm{H})$ holds. Then problem $\left(1_{\bar{\mu}}\right)$ has a solution.

Proof. Let $v_{\mu}$ be the function introduced in the proof of Lemma 3.3. The function $v_{\mu}$ satisfies

$$
\begin{cases}-\Delta v_{\mu}=Q(x)\left(v_{\mu}+\mu\right)^{p} & \text { in } \Omega^{c}, \\ \frac{\partial v_{\mu}}{\partial \nu}=0 & \text { on } \partial \Omega, \\ \lim _{|x| \rightarrow \infty} v_{\mu}(x)=0 . & \end{cases}
$$

We commence by showing that

$$
\int_{\Omega^{c}} v_{\mu}^{q} d x \leq C
$$

for some constant $C>0$ independent of $\mu$ and for all $q \geq 2^{*}$. Due to the estimates of Lemma $2.1 \phi_{j}\left(v_{\mu}\right) \in D^{1,2}\left(\Omega^{c}\right)$, where $\phi_{j}(t)=t^{j}, j \geq 1$. It follows from Lemma 3.2 that

$$
\left.\int_{\Omega^{c}}\left|\phi_{j}^{\prime}\left(v_{\mu}\right)^{2}\right| \nabla v_{\mu}\right|^{2} d x \geq p \int_{\Omega^{c}} Q(x)\left(v_{\mu}+\mu\right)^{p-1} \phi_{j}\left(v_{\mu}\right)^{2} d x .
$$

Let $\psi_{j}(t)=\int_{0}^{t} \phi_{j}^{\prime}(s)^{2} d s=j^{2} /(2 j-1) t^{2 j-1}$. Testing (3.7) with $\psi_{j}\left(v_{\mu}\right)$ we get

$$
\int_{\Omega^{c}} \psi_{j}^{\prime}\left(v_{\mu}\right)\left|\nabla v_{\mu}\right|^{2} d x=\int_{\Omega^{c}} Q(x)\left(v_{\mu}+\mu\right)^{p} \psi_{j}\left(v_{\mu}\right) d x .
$$


We deduce from (3.9) and (3.10) that

$$
\begin{aligned}
p \int_{\Omega^{c}} Q(x)\left(v_{\mu}+\mu\right)^{p-1} v_{\mu}^{2 j} d x \leq \frac{j^{2}}{2 j-1}\left[\int_{\Omega^{c}} Q(x)\left(v_{\mu}+\mu\right)^{p-1} v_{\mu}^{2 j} d x\right. \\
\left.+\int_{\Omega^{c}} Q(x)\left(v_{\mu}+\mu\right)^{p-1} \mu v_{\mu}^{2 j-1}\right] .
\end{aligned}
$$

We now choose $j_{\circ}>1$, close to 1 , so that $j^{2} /(2 j-1)<p$ for every $j \leq j_{\circ}$. Let $p-j^{2} /(2 j-1)=\alpha(j, p)>0$. We then derive from the above estimate that

$$
\begin{array}{rl}
\alpha(j, p) \int_{\Omega^{c}} & Q(x) v_{\mu}^{p+2 j-1} d x \leq \alpha(j, p) \int_{\Omega^{c}} Q(x)\left(v_{\mu}+\mu\right)^{p-1} v_{\mu}^{2 j} d x \\
& \leq \frac{j^{2}}{2 j-1} \int_{\Omega^{c}} Q(x)\left(v_{\mu}+\mu\right)^{p-1} \mu v_{\mu}^{2 j-1} d x \\
& \leq \frac{C j^{2}}{2 j-1}\left[\int_{\Omega^{c}} Q(x) v_{\mu}^{p+2 j-2} \mu d x+\int_{\Omega^{c}} Q(x) \mu^{p} v_{\mu}^{2 j-1} d x\right] \\
& \leq C\left[\int_{\Omega^{c}} Q(x) v_{\mu}^{p+2 j-2} d x+\int_{\Omega^{c}} Q(x) v_{\mu}^{2 j-1} d x\right]
\end{array}
$$

where $C=C(\bar{\mu}, j)$. We now estimate both integrals on the right side of this inequality. By the Hölder and Young inequalities we have for every $\delta>0$

$$
\begin{aligned}
\int_{\Omega^{c}} Q(x) v_{\mu}^{p+2 j-2} d x \leq & \left(\int_{\Omega^{c}} Q(x) d x\right)^{1 /(p+2 j-1)} \\
& \cdot\left(\int_{\Omega^{c}} Q(x) v_{\mu}^{p+2 j-1} d x\right)^{(p+2 j-2) /(p+2 j-1)} \\
\leq & \frac{\delta}{2} \int_{\Omega^{c}} Q(x) v_{\mu}^{p+2 j-1} d x+C(\delta) \int_{\Omega^{c}} Q(x) d x
\end{aligned}
$$

For the second integral we have

$$
\int_{\Omega^{c}} Q(x) v_{\mu}^{2 j-1} d x \leq \frac{\delta}{2} \int_{\Omega^{c}} Q(x) v_{\mu}^{p+2 j-1} d x+C(\delta) \int_{\Omega^{c}} Q(x) .
$$

It then follows from (3.11) and the last two estimates

$$
\int_{\Omega^{c}} Q(x) v_{\mu}^{p+2 j-1} d x \leq C_{1}(\delta)
$$

for some $\delta>0$ small enough with a constant $C_{1}(\delta)$ independent of $\mu$. Combining (3.10), (3.12), (3.13) and the Sobolev inequality we get

$$
\left(\int_{\Omega^{c}} v_{\mu}^{j(p+1)} d x\right)^{(N-2) / N} \leq C_{1} \int_{\Omega^{c}} Q(x) v_{\mu}^{p+2 j-1} d x+C_{2}
$$

for some constant $C_{1}>0$ and $C_{2}>0$ independent of $\mu$. We choose $2 N /(N-2)<$ $q \leq p+2 j_{\circ}-1$ and write it as $q=(p+1) j$ for some $1<j \leq j_{\circ}$. Therefore we 
have

$$
\int_{\Omega^{c}} v_{\mu}^{q} d x \leq C
$$

for some constant $C$ independent of $\mu \in(0, \bar{\mu})$ and for every $p+1 \leq q \leq p+2 j_{\circ}-1$. We now take $q_{\circ}=p+1=2 N /(N-2)$ and $\delta=p+2 j_{\circ}-1-2 N /(N-2)>0$.

Testing (3.7) with $v_{\mu}^{q_{\circ}-1}$ we get

$$
\begin{aligned}
\frac{4\left(q_{\circ}-1\right)}{q_{\circ}} \int_{\Omega^{c}}\left|\nabla v_{\mu}^{q_{\circ} / 2}\right|^{2} d x=\int_{\Omega^{c}} Q(x)\left(v_{\mu}+\mu\right)^{p} v_{\mu}^{q_{\circ}-1} d x \\
\leq C\left[\int_{\Omega^{c}} Q(x) v_{\mu}^{p+q_{\circ}-1} d x+\int_{\Omega^{c}} Q(x) v_{\mu}^{q_{\circ}-1} d x\right] \\
\leq C \int_{\Omega^{c}} Q(x) v_{\mu}^{p+q_{\circ}-1} d x \\
+C\left(\int_{\Omega^{c}} Q(x) v_{\mu}^{p+q_{\circ}-1} d x\right)^{\left(q_{\circ}-1\right) /\left(p+q_{\circ}-1\right)} \\
\\
\cdot\left(\int_{\Omega^{c}} Q(x) d x\right)^{p /\left(p+q_{\circ}-1\right)} \\
\leq C_{1} \int_{\Omega^{c}} Q(x) v_{\mu}^{p+q_{\circ}-1} d x+C_{2} \int_{\Omega^{c}} Q(x) d x,
\end{aligned}
$$

where $C_{1}>0$ and $C_{2}>0$ are constants independent of $\mu$. Since $q_{\circ}<q_{\circ}+p-1<$ $p-1+q_{\circ}+2 \delta / N$, we have

$$
t^{p-1+q_{\circ}} \leq \varepsilon t^{p-1+q_{\circ}+2 \delta / N}+C_{\varepsilon} t^{q \circ}
$$

for every $t \geq 0$. Applying (3.15) with $q=p+2 j_{\circ}-1$, we get

$$
\begin{aligned}
& \int_{\Omega^{c}} Q(x) v_{\mu}^{p+q_{\circ}-1} d x \leq \varepsilon \int_{\Omega^{c}} Q(x) v_{\mu}^{p-1+q_{\circ}+2 \delta / N} d x+C_{\varepsilon} \\
& \leq \varepsilon\left(\int_{\Omega^{c}} Q(x)\left(v_{\mu}^{q_{\circ}}\right)^{(p+1) / 2} d x\right)^{2 /(p+1)}\left(\int_{\Omega^{c}} Q(x) v_{\mu}^{(p-1+2 \delta / N) N / 2} d x\right)^{2 / N}+C \\
& \leq \varepsilon C \int_{\Omega^{c}} Q(x)\left(v_{\mu}\right)^{q_{\circ}(p+1) / 2} d x+C_{1} \leq \varepsilon C_{2} \int_{\Omega^{c}}\left|\nabla v_{\mu}^{q_{\circ} / 2}\right|^{2} d x+C_{3} .
\end{aligned}
$$

This combined with (3.16) gives

$$
\int_{\Omega^{c}}\left|\nabla v_{\mu}^{q_{\circ} / 2}\right|^{2} d x \leq C
$$

for some $C>0$ independent of $\mu$. By the Sobolev inequality we get

$$
\int_{\Omega^{c}} v_{\mu}^{q_{o}^{2} / 2} d x \leq C
$$

and the result follows by iteration.

It follows from (3.8) that $Q\left(v_{\mu}+\mu\right) \in L^{q}\left(\Omega^{c}\right)$ for every $q \geq p+1$. Therefore using the $L^{p}$ estimates up to the boundary [1] and the interior $L^{p}$ estimates ([8, 
Theorem 9.11]), we show as in [6] that up to a subsequence, $v_{\mu} \rightarrow v$ as $\mu \rightarrow \bar{\mu}$ in $C^{1}\left(\bar{\Omega}^{c} \cap B(0, R)\right)$ for all $R>0$. Due to Lemma 3.3 we can also assume that $v \in D^{1,2}\left(\Omega^{c}\right)$ and $v$ is a weak solution of

$$
\begin{cases}-\Delta v=Q(x)(v+\bar{\mu})^{p} & \text { in } \Omega^{c} \\ \frac{\partial v_{\mu}}{\partial \nu}=0 & \text { on } \partial \Omega .\end{cases}
$$

By the results of the next section $\lim _{|x| \rightarrow \infty} v(x)=0$. Thus $v+\bar{\mu}$ is a solution of problem $\left(1_{\bar{\mu}}\right)$. The solution $u_{\bar{\mu}}$ is unique. Indeed, let $\widetilde{u}_{\bar{\mu}}$ be another solution of $\left(1_{\bar{\mu}}\right)$. Since $\widetilde{u}_{\bar{\mu}}$ is a supersolution of $\left(1_{\mu}\right)$ for $\mu<\bar{\mu}$, we see that $\widetilde{u}_{\bar{\mu}}>u_{\mu}$ for $\mu<\bar{\mu}$. Consequently, $\widetilde{u}_{\bar{\mu}} \geq u_{\bar{\mu}}$. We now show that $\sigma_{\bar{\mu}}=1$. Otherwise, applying the implicit function theorem to the operator $F(v, \mu)=-\Delta v+Q(x)\left(v^{\mu}\right)^{p}$ as a mapping from $D^{1,2}\left(\Omega^{c}\right) \times[0, \infty)$ into $D^{1,2}\left(\Omega^{c}\right)$, we deduce the existence of a positive solution $v$ for every $\mu$ in a small interval $(\bar{\mu}-\delta, \bar{\mu}+\delta)$. By the results of the next section these solutions have limit equal to 0 as $|x| \rightarrow \infty$. Clearly, this contradicts the definition of $\bar{\mu}$. Repeating the argument from p. 216 of [6] we show that $\widetilde{u}_{\bar{\mu}}=u_{\bar{\mu}}$.

\section{Application of the mountain-pass principle}

For every $\mu \in(0, \bar{\mu})$ we consider the problem

$$
\begin{cases}-\Delta v=Q(x)\left(\left(v+u_{\mu}\right)^{2^{*}-1}-u_{\mu}^{2^{*}-1}\right) & \text { in } \Omega^{c} \\ \frac{\partial v}{\partial \nu}=0 & \text { on } \partial \Omega \\ v>0 & \text { on } \Omega^{c} \\ \lim _{|x| \rightarrow \infty} v(x)=0, & \end{cases}
$$

where $u_{\mu}$ is a minimal solution of $\left(1_{\mu}\right)$. If $v_{\mu}$ is a solution of $(4.1)$, then $U_{\mu}=$ $v_{\mu}+u_{\mu}$ is a solution of $\left(1_{\mu}\right)$. A solution of (4.1) will be found as a critical point of the functional

$$
\begin{aligned}
J_{\mu}(v)= & \frac{1}{2} \int_{\Omega^{c}}|\nabla v|^{2} d x-\frac{1}{2^{*}} \int_{\Omega^{c}} Q(x)\left(u_{\mu}+v^{+}\right)^{2^{*}} d x \\
& +\frac{1}{2^{*}} \int_{\Omega^{c}} Q(x) u_{\mu}^{2^{*}} d x+\int_{\Omega^{c}} Q(x) u_{\mu}^{2^{*}-1} v^{+} d x
\end{aligned}
$$

for $v \in D^{1,2}\left(\Omega^{c}\right)$. It is easy to show that $J_{\mu}$ is a $C^{1}$-functional and we have

$$
\left\langle J_{\mu}^{\prime}(v), \phi\right\rangle=\int_{\Omega^{c}}\left[\nabla v \nabla \phi-Q(x)\left(\left(u_{\mu}+v^{+}\right)^{2^{*}-1}-u_{\mu}^{2^{*}-1}\right)\right] \phi d x
$$

for every $\phi \in D^{1,2}\left(\Omega^{c}\right)$. To show that the functional $J_{\mu}$ has a mountain-pass structure, we need the following inequality: let $p>2$, then for every $\varepsilon>0$ there exists $C_{\varepsilon}>0$ such that, for every $s \geq 0$,

$$
\left(u_{\mu}+s\right)^{p}-u_{\mu}^{p}-p u_{\mu}^{p-1} s \leq \varepsilon u_{\mu}^{p-1} s+C_{\varepsilon} s^{p} .
$$


Lemma 4.1. There exist $\alpha>0$ and $\rho>0$ such that $J_{\mu}(v) \geq \alpha>0$ for $v \in D^{1,2}\left(\Omega^{c}\right)$ with $\|\nabla v\|_{2}=\rho$.

Proof. We write $J_{\mu}$ in the form

$$
\begin{aligned}
J_{\mu}(v)= & \frac{1}{2} \int_{\Omega^{c}}|\nabla v|^{2} d x-\frac{2^{*}-1}{2} \int_{\Omega^{c}} Q(x) u_{\mu}^{2^{*}-2}\left(v^{+}\right)^{2} d x \\
& -\int_{\Omega^{c}} \int_{0}^{v^{+}} Q(x)\left[\left(u_{\mu}+s\right)^{2^{*}-1}-u_{\mu}^{2^{*}-1}-\left(2^{*}-1\right) u_{\mu}^{2^{*}-2} s\right] d s d x .
\end{aligned}
$$

Applying (4.2) with $p=2^{*}-1$ we get

$$
\begin{aligned}
J_{\mu}(v) \geq & \frac{1}{2} \int_{\Omega^{c}}\left[|\nabla v|^{2}-\left(2^{*}-1\right) Q(x) u_{\mu}^{2^{*}-2}\left(v^{+}\right)^{2}\right] d x \\
& -\int_{\Omega^{c}} Q(x)\left[\frac{\varepsilon}{2} u_{\mu}^{2^{*}-2}\left(v^{+}\right)^{2}+C_{\varepsilon} \frac{\left(v^{+}\right)^{2^{*}}}{2^{*}}\right] d x .
\end{aligned}
$$

Hence by Lemma 3.2 we have

$$
J_{\mu}(v) \geq \frac{1}{2}\left(1-\frac{2^{*}-1-\varepsilon}{\sigma_{\mu}\left(2^{*}-1\right)}\right) \int_{\Omega^{c}}|\nabla v|^{2} d x-\frac{C_{\varepsilon}}{2^{*}} \int_{\Omega^{c}} Q(x) \frac{\left(v^{+}\right)^{2^{*}}}{2^{*}} d x .
$$

An application of the Sobolev inequality completes the proof.

In Propositions 4.2 and 4.3, below, we examine the (PS) sequences of the functional $J_{\mu}$.

Proposition 4.2. Let $\left\{v_{m}\right\} \subset D^{1,2}\left(\Omega^{c}\right)$ be a $(\mathrm{PS})_{c}$ sequence for $J_{\mu}$. Then $\left\{v_{m}\right\}$ is bounded in $D^{1,2}\left(\Omega^{c}\right)$.

Proof. We compute

$$
\begin{aligned}
J_{\mu}\left(v_{m}\right) & -\frac{1}{2}\left\langle J_{\mu}^{\prime}\left(v_{m}\right), v_{m}\right\rangle \\
= & -\frac{1}{2^{*}} \int_{\Omega^{c}} Q(x)\left(u_{\mu}+v_{m}^{+}\right)^{2^{*}} d x+\frac{1}{2^{*}} \int_{\Omega^{c}} Q(x) u_{\mu}^{2^{*}} d x \\
& +\int_{\Omega^{c}} Q(x) u_{\mu}^{2^{*}-1} v_{m}^{+} d x+\frac{1}{2} \int_{\Omega^{c}} Q(x)\left(u_{\mu}+v_{m}^{+}\right)^{2^{*}-1} v_{m} d x \\
& -\frac{1}{2} \int_{\Omega^{c}} Q(x) u_{\mu}^{2^{*}-1} v_{m} d x \\
= & \frac{1}{N} \int_{\Omega^{c}} Q(x)\left(u_{\mu}+v_{m}^{+}\right)^{2^{*}} d x-\frac{1}{2} \int_{\Omega^{c}} Q(x)\left(u_{\mu}+v_{m}^{+}\right)^{2^{*}-1} v_{m}^{-} d x \\
& -\frac{1}{2} \int_{\Omega^{c}} Q(x)\left(u_{\mu}+v_{m}^{+}\right)^{2^{*}-1} u_{\mu} d x+\frac{1}{2^{*}} \int_{\Omega^{c}} Q(x) u_{\mu}^{2^{*}} d x \\
& +\int_{\Omega^{c}} Q(x) u_{\mu}^{2^{*}-1} v_{m}^{+} d x-\frac{1}{2} \int_{\Omega^{c}} Q(x) u_{\mu}^{2^{*}-1} v_{m} d x \\
= & \frac{1}{N} \int_{\Omega^{c}} Q(x)\left(u_{\mu}+v_{m}^{+}\right)^{2^{*}} d x-\frac{1}{2} \int_{\Omega^{c}} Q(x)\left(u_{\mu}+v_{m}^{+}\right)^{2^{*}-1} u_{\mu} d x
\end{aligned}
$$




$$
\begin{aligned}
& \quad-\frac{1}{2} \int_{\Omega^{c}} Q(x) u_{\mu}^{2^{*}-1} v_{m}^{-} d x+\frac{1}{2^{*}} \int_{\Omega^{c}} Q(x) u_{\mu}^{2^{*}} d x \\
& +\int_{\Omega^{c}} Q(x) u_{\mu}^{2^{*}-1} v_{m}^{+} d x-\frac{1}{2} \int_{\Omega^{c}} Q(x) u_{\mu}^{2^{*}-1} v_{m} d x \\
& =\frac{1}{N} \int_{\Omega^{c}} Q(x)\left(u_{\mu}+v_{m}^{+}\right)^{2^{*}} d x-\frac{1}{2} \int_{\Omega^{c}} Q(x)\left(u_{\mu}+v_{m}^{+}\right)^{2^{*}-1} u_{\mu} d x \\
& \quad+\frac{1}{2} \int_{\Omega^{c}} Q(x) u_{\mu}^{2^{*}-1} v_{m}^{+} d x+\frac{1}{2^{*}} \int_{\Omega^{c}} Q(x) u_{\mu}^{2^{*}} d x .
\end{aligned}
$$

Given $\delta>0$ we choose $C(\delta)>0$ that

$\int_{\Omega^{c}} Q(x)\left(u_{\mu}+v_{m}^{+}\right)^{2^{*}-1} u_{\mu} d x \leq \delta \int_{\Omega^{c}} Q(x)\left(u_{\mu}+v_{m}^{+}\right)^{2^{*}} d x+C(\delta) \int_{\Omega^{c}} Q(x) u_{\mu}^{2^{*}} d x$.

Taking $\delta>0$ small and using the fact that $\left\{v_{m}\right\}$ is a $(\mathrm{PS})_{c}$ sequence we deduce from (4.3) that there exist constants $C_{1}>0$ and $C_{2}>0$ such that

$$
\int_{\Omega^{c}} Q(x)\left(u_{\mu}+v_{m}^{+}\right)^{2^{*}} d x \leq C_{1}+C_{2}\left\|\nabla v_{m}\right\|_{2}
$$

for every $m \geq 1$. On the other hand we have

$$
\begin{aligned}
J_{\mu}\left(v_{m}\right) & -\frac{1}{2^{*}}\left\langle J_{\mu}^{\prime}\left(v_{m}\right), v_{m}\right\rangle=\frac{1}{N} \int_{\Omega^{c}}\left|\nabla v_{m}\right|^{2} d x \\
& +\frac{1}{2^{*}} \int_{\Omega^{c}} Q(x)\left(u_{\mu}+v_{m}^{+}\right)^{2^{*}-1}\left(v_{m}-v_{m}^{+}-u_{\mu}\right) d x+\frac{1}{2^{*}} \int_{\Omega^{c}} Q(x) u_{\mu}^{2^{*}} d x \\
& +\int_{\Omega^{c}} Q(x) u_{\mu}^{2^{*}-1} v_{m}^{+} d x-\frac{1}{2^{*}} \int_{\Omega^{c}} Q(x) u_{\mu}^{2^{*}-1} v_{m} d x \\
= & \frac{1}{N} \int_{\Omega^{c}}\left|\nabla v_{m}\right|^{2} d x-\frac{1}{2^{*}} \int_{\Omega^{c}} Q(x)\left(u_{\mu}+v_{m}^{+}\right)^{2^{*}-1} u_{\mu} d x \\
& -\frac{1}{2^{*}} \int_{\Omega^{c}} Q(x)\left(u_{\mu}+v_{m}^{+}\right)^{2^{*}-1} v_{m}^{-} d x \\
& +\frac{1}{2^{*}} \int_{\Omega^{c}} Q(x) u_{\mu}^{2^{*}} d x+\int_{\Omega^{c}} Q(x) u_{\mu}^{2^{*}-1} v_{m}^{+} d x-\frac{1}{2^{*}} \int_{\Omega^{c}} Q(x) u_{\mu}^{2^{*}-1} v_{m} d x \\
= & \frac{1}{N} \int_{\Omega^{c}}\left|\nabla v_{m}\right|^{2} d x-\frac{1}{2^{*}} \int_{\Omega^{c}} Q(x)\left(u_{\mu}+v_{m}^{+}\right)^{2^{*}-1} u_{\mu} d x \\
& +\frac{1}{2^{*}} \int_{\Omega^{c}} Q(x) u_{\mu}^{2^{*}} d x+\left(1-\frac{1}{2^{*}}\right) \int_{\Omega^{c}} Q(x) u_{\mu}^{2^{*}-1} v_{m}^{+} d x \\
\geq & \frac{1}{N} \int_{\Omega^{c}}\left|\nabla v_{m}\right|^{2} d x-\frac{1}{2^{*}} \int_{\Omega^{c}} Q(x)\left(u_{\mu}+v_{m}^{+}\right)^{2^{*}-1} u_{\mu} d x .
\end{aligned}
$$

From this we derive, using the Young inequality, that

$$
\left\|\nabla v_{m}\right\|_{2}^{2} \leq C_{3} \int_{\Omega^{c}} Q(x)\left(u_{\mu}+v_{m}^{+}\right)^{2^{*}} d x+C_{4}\left\|\nabla v_{m}\right\|_{2}+C_{5} .
$$

The fact that $\left\{v_{m}\right\}$ is a bounded sequence in $D^{1,2}\left(\Omega^{c}\right)$ is a consequence of (4.4) and (4.5). 
To proceed further we set

$$
Q_{m}=\max _{x \in \partial \Omega} Q(x) \quad \text { and } \quad Q_{M}=\max _{x \in \Omega^{c}} Q(x) .
$$

These two quantities play an essential role in finding an energy level of the functional $J_{\mu}$ below which the Palais-Smale condition holds (see also [4] and [5]).

Proposition 4.3. Suppose that

$$
J_{\mu}\left(v_{m}\right) \rightarrow c<\min \left(\frac{S^{N / 2}}{2 N Q_{m}^{(N-2) / 2}}, \frac{S^{N / 2}}{N Q_{M}^{(N-2) / 2}}\right), \quad c>0,
$$

and

$$
J_{\mu}^{\prime}\left(v_{m}\right) \rightarrow 0 \quad \text { in } D^{-1,2}\left(\Omega^{c}\right)
$$

Then the sequence $\left\{v_{m}\right\}$ has a subsequence converging weakly in $D^{1,2}\left(\Omega^{c}\right)$ to a non zero limit.

Proof. Since by Proposition $4.2\left\{v_{m}\right\}$ is bounded in $D^{1,2}\left(\Omega^{c}\right)$, we may assume that $v_{m} \rightarrow v$ in $D^{1,2}\left(\Omega^{c}\right)$ and $v_{m} \rightarrow v$ in $L^{p}\left(\Omega^{c}\right) \cap B(0, R)$ ) for each $2 \leq p<2^{*}$ and $R>0$ with $\Omega \subset B(0, R)$. Testing (4.7) with $\phi=v_{m}^{-}$we get that

$$
\int_{\Omega^{c}}\left|\nabla v_{m}^{-}\right|^{2} d x=o(1)
$$

Therefore we may assume that $v_{m} \geq 0$ on $\Omega^{c}$. We now show that $v \not \equiv 0$. Arguing, by contradiction assume that $v=0$ on $\Omega^{c}$. We must have $v_{m} \nrightarrow 0$ in $D^{1,2}\left(\Omega^{c}\right)$ because $c>0$. Hence the sequence $\left\{v_{m}\right\}$ must concentrate. It cannot concentrate at infinity since $Q(x) \rightarrow 0$ as $|x| \rightarrow \infty$. Therefore the concentration occurs either on $\partial \Omega$ or inside $\Omega$. By the P. L. Lions concentration-compactness principle (see [10]), there exist sequences of points $\left\{x_{j}\right\} \subset \mathbb{R}^{N}$ and numbers $\left\{\nu_{j}\right\}$, $\left\{\mu_{j}\right\} \subset(0, \infty)$ such that

$$
\left|v_{m}\right|^{2^{*}} \stackrel{*}{\rightarrow} \sum_{j} \nu_{j} \delta_{j} \quad \text { and } \quad\left|\nabla v_{m}\right|^{2} \stackrel{*}{\rightarrow} \sum_{j} \mu_{j} \delta_{j}
$$

in $\mathcal{M}$, where $\mathcal{M}$ is a space of measures, moreover

$$
\begin{gathered}
S \nu_{j}^{2 / 2^{*}} \leq \mu_{j} \quad \text { if } x_{j} \in \Omega, \\
S \frac{\nu_{j}^{2 / 2^{*}}}{2^{2 / N}} \leq \mu_{j} \quad \text { if } x_{j} \in \partial \Omega .
\end{gathered}
$$

From (4.7) we deduce that $\mu_{j} \leq Q\left(x_{j}\right) \nu_{j}$ for every $j$. If $\nu_{j}>0$ and $x_{j} \in \Omega$, then $\nu_{j} \geq S^{N / 2} / Q\left(x_{j}\right)^{N / 2}$ and if $x_{j} \in \partial \Omega$, then $\nu_{j} \geq S^{N / 2} /\left(2 Q\left(x_{j}\right)^{N / 2}\right)$. By the 
Brézis-Lieb lemma (see [3]) we have

$$
\begin{aligned}
J_{\mu}\left(v_{m}\right) & -\frac{1}{2}\left\langle J_{\mu}^{\prime}\left(v_{m}\right), v_{m}\right\rangle=\frac{1}{N} \int_{\Omega^{c}} Q(x)\left(u_{\mu}+v_{m}\right)^{2^{*}} d x \\
& -\frac{1}{2} \int_{\Omega^{c}} Q(x)\left(u_{\mu}+v_{m}\right)^{2^{*}-1} u_{\mu} d x+\frac{1}{2^{*}} \int_{\Omega^{c}} Q(x) u_{\mu}^{2^{*}} d x+o(1) \\
= & \frac{1}{N} \int_{\Omega^{c}} Q(x) u_{\mu}^{2^{*}} d x+\frac{1}{N} \int_{\Omega^{c}} Q(x) v_{m}^{2^{*}} d x \\
& -\frac{1}{2} \int_{\Omega^{c}} Q(x) u_{\mu}^{2^{*}} d x+\frac{1}{2^{*}} \int_{\Omega^{c}} Q(x) u_{\mu}^{2^{*}} d x+o(1) \\
= & \frac{1}{N} \int_{\Omega^{c}} Q(x) v_{m}^{2^{*}} d x+o(1) \\
= & \frac{1}{N} \sum_{x_{j} \in \partial \Omega} Q\left(x_{j}\right) \nu_{j}+\frac{1}{N} \sum_{x_{j} \in \Omega} Q\left(x_{j}\right) \nu_{j}+o(1) \\
\geq & \frac{1}{N} \sum_{x_{j} \in \partial \Omega} \frac{S^{N / 2}}{Q\left(x_{j}\right)^{(N-2) / 2}}+\frac{1}{N} \sum_{x_{j} \in \Omega} \frac{S^{N / 2}}{Q\left(x_{j}\right)^{(N-2) / 2}}+o(1) .
\end{aligned}
$$

If $Q_{M}>2^{2 /(N-2)} Q_{m}$, then letting $m \rightarrow \infty$ we derive that $c \geq S^{N / 2} /\left(N Q_{M}^{(N-2) / 2}\right)$ and if $Q_{M} \leq 2^{2 /(N-2)} Q_{m}$, then $c \geq S^{N / 2} /\left(2 N Q_{m}^{(N-2) / 2}\right)$. In both cases we get a contradiction.

LEMma 4.4. There exists $\psi_{\circ} \in D^{1,2}\left(\Omega^{c}\right)$ such that $\left\|\nabla \psi_{\circ}\right\|_{2}>\rho$ and $J_{\mu}\left(\psi_{\circ}\right)<0$, where $\rho>0$ is a constant from Lemma 4.1 .

Proof. Let $\phi_{\circ} \in D^{1,2}\left(\Omega^{c}\right)$ and $\phi_{\circ}>0$ on $\Omega^{c}$. We then have for $\psi_{\circ}=t \phi_{\circ}$

$$
\begin{aligned}
J_{\mu}\left(t \phi_{\circ}\right) \leq & \frac{t^{2}}{2} \int_{\Omega^{c}}\left|\nabla \phi_{\circ}\right|^{2} d x-\frac{t^{2^{*}}}{2^{*}} \int_{\Omega^{c}} Q(x) \phi_{\circ}^{2^{*}} d x \\
& +\frac{1}{2^{*}} \int_{\Omega^{c}} Q(x) u_{\mu}^{2^{*}} d x+t \int_{\Omega^{c}} Q(x) u_{\mu}^{2^{*}-1} \phi_{\circ} d x<0
\end{aligned}
$$

for $t>0$ sufficiently large.

To apply the mountain-pass principle we define

$$
c=\inf _{\gamma \in \Gamma} \max _{t \in[0,1]} J_{\mu}(\gamma(t)),
$$

where $\Gamma=\left\{\gamma: \gamma \in C\left([0,1], D^{1,2}\left(\Omega^{c}\right)\right), \gamma(0)=0, \gamma(1)=\psi_{\circ}\right\}$.

THEOREM 4.5.

(a) Let $Q_{M} \leq 2^{2 /(N-2)} Q_{m}$. Suppose that $|Q(x)-Q(y)|=o(|x-y|)$ for $x$ close to $y, Q(y)=Q_{m}$ and with the mean curvature $H(y)<0$ when viewed from inside $\Omega$. Then problem (4.1) has a solution.

(b) Let $Q_{M}>2^{2 /(N-2)} Q_{m}$. Suppose that $|Q(x)-Q(y)|=o\left(|x-y|^{N-2}\right)$ for $x$ close to $y$ with $Q(y)=Q_{M}$. Then problem (4.1) has a solution. 
Proof. Since

$$
\int_{\Omega^{c}} Q(x) \int_{0}^{v+}\left[\left(u_{\mu}+s\right)^{2^{*}-1}-u_{\mu}^{2^{*}-1}-s^{2^{*}-1}\right] d s d x \geq 0
$$

we have

$$
\begin{aligned}
J_{\mu}(v)= & \frac{1}{2} \int_{\Omega^{c}}|\nabla v|^{2} d x-\frac{1}{2^{*}} \int_{\Omega^{c}} Q(x)\left(u_{\mu}+v^{+}\right)^{2^{*}} d x \\
& +\frac{1}{2^{*}} \int_{\Omega^{c}} Q(x) u_{\mu}^{2^{*}} d x+\int_{\Omega^{c}} Q(x) u_{\mu}^{2^{*}-1} v^{+} d x \\
= & \frac{1}{2} \int_{\Omega^{c}}|\nabla v|^{2} d x-\frac{1}{2^{*}} \int_{\Omega^{c}} Q(x)\left(v^{+}\right)^{2^{*}} d x \\
& -\int_{\Omega^{c}} Q(x) \int_{0}^{v+}\left[\left(u_{\mu}+s\right)^{2^{*}-1}-u_{\mu}^{2^{*}-1}-s^{2^{*}-1}\right] d s d x \\
\leq & \frac{1}{2} \int_{\Omega^{c}}|\nabla v|^{2} d x-\frac{1}{2^{*}} \int_{\Omega^{c}} Q(x)\left(v^{+}\right)^{2^{*}} d x .
\end{aligned}
$$

Hence

$$
\begin{aligned}
\max _{t \geq 0} J_{\mu}(t v) & \leq \max _{t \geq 0}\left(\frac{t^{2}}{2} \int_{\Omega^{c}}|\nabla v|^{2} d x-\frac{t^{2^{*}}}{2^{*}} \int_{\Omega^{c}} Q(x)\left(v^{+}\right)^{2^{*}} d x\right) \\
& =\frac{1}{N} \frac{\left(\int_{\Omega^{c}}|\nabla v|^{2} d x\right)^{N / 2}}{\left(\int_{\Omega^{c}} Q(x)\left(v^{+}\right)^{2^{*}} d x\right)^{(N-2) / 2}} .
\end{aligned}
$$

(a) We consider the case $Q_{M} \leq 2^{2 /(N-2)} Q_{m}$. Let

$$
\begin{aligned}
U_{\varepsilon, y}(x)=\varepsilon^{-(N-2) / 2} U\left(\frac{x-y}{\varepsilon}\right), & \varepsilon>0, y \in \mathbb{R}^{N} \\
& \text { where } U(x)=\frac{[N(N-2)]^{(N-2) / 2}}{\left(N(N-2)+|x|^{2}\right)^{(N-2) / 2}} .
\end{aligned}
$$

This function, called an instanton, has a property

$$
\int_{\mathbb{R}^{N}}\left|\nabla U_{\varepsilon, y}\right|^{2} d x=\int_{\mathbb{R}^{N}} U_{\varepsilon, y}^{2^{*}} d x=S^{N / 2} .
$$

Moreover, it is known that

$$
\frac{\int_{\Omega^{c}}|\nabla v|^{2} d x}{\left(\int_{\Omega^{c}} U_{\varepsilon, y}^{2^{*}} d x\right)^{2 / 2^{*}}}=\frac{S}{2^{2 / N}}+ \begin{cases}A_{N} H(y) \varepsilon \log (1 / \varepsilon)+O(\varepsilon) & \text { for } N=3, \\ A_{N} H(y) \varepsilon+O\left(\varepsilon^{2} \log (1 / \varepsilon)\right. & \text { for } N=4, \\ A_{N} H(y) \varepsilon+O\left(\varepsilon^{2}\right) & \text { for } N=5,\end{cases}
$$

where $A_{N}>0$ is a constant depending on $N$. This estimate can be obtained from the corresponding estimate on a bounded domain by truncation (see [2], [11], [9]). Substituting $v=U_{\varepsilon, y}$ in (4.8) and using the above estimate together with our assumption $Q$ we get the following estimate for the mountain-pass level

$$
c<\frac{S^{N / 2}}{2 N Q_{m}^{N-2 / 2}}
$$


(b) If $Q_{M}>2^{2 /(N-2)} Q_{m}$, we take $U_{\varepsilon, y}$ with $Q(y)=Q_{M}$. We then have

$$
\int_{\Omega^{c}}\left|\nabla U_{\varepsilon, y}\right|^{2} d x=\int_{\mathbb{R}^{N}}|\nabla U|^{2} d x-\int_{\Omega}\left|\nabla U_{\varepsilon, y}\right|^{2} d x \leq S^{N / 2}-C_{1} \varepsilon^{N-2}
$$

for some constant $C_{1}>0$ and

$$
\begin{aligned}
\int_{\Omega^{c}} Q(x) U_{\varepsilon, y}^{2^{*}} d x= & \int_{\Omega^{c}} Q_{M} U_{\varepsilon, y}^{2^{*}} d x+\int_{\Omega^{c}}\left(Q(x)-Q_{M}\right) U_{\varepsilon, y}^{2^{*}} d x \\
& =S^{N / 2} Q_{M}+o\left(\varepsilon^{N-2}\right) .
\end{aligned}
$$

Using the last two relations in (4.8) we see that

$$
c<\frac{S^{N / 2}}{N Q_{M}^{N-2 / 2}} .
$$

\section{Main result}

To use a solution $u$ of problem (4.1) to construct a second solution of $\left(1_{\mu}\right)$ we have to show that $\lim _{|x| \rightarrow \infty} u(x)=0$. This will be accomplished by using the Moser iteration technique. In Proposition 5.1 below, we use some ideas from the proof of Theorem 8.17 in [8].

Proposition 5.1. Suppose that $\lim _{|x| \rightarrow \infty} Q(x)=0$ and $Q \in L^{N / 2}\left(\Omega^{c}\right)$. Let $u \in D^{1,2}\left(\Omega^{c}\right)$ be a positive solution of (4.1). Then there exists $R>0$ such that for every $B\left(x_{\circ}, 2\right) \subset(|x|>R)$ we have

$$
\sup _{B\left(x_{\circ}, 1\right)} u(x) \leq C\left(\int_{B\left(x_{\circ}, 2\right)} u^{2^{*}} d x\right)^{1 / 2^{*}},
$$

where a constant $C$ depends on $u$ but is independent of $x_{0}$.

Proof. Let $\varepsilon>0$ be fixed and set $p=2^{*}-1$. We choose a constant $C_{\varepsilon}>0$ such that

$$
\left(u+u_{\mu}\right)^{p}-u_{\mu}^{p} \leq(p+\varepsilon) u_{\mu}^{p-1} u+C_{\varepsilon} u^{p}
$$

for every $x \in \Omega^{c}$. Then

$$
-\Delta u \leq d(x) u \quad \text { on } \Omega^{c},
$$

where $d(x)=Q(x)(p+\varepsilon) u_{\mu}^{p-1}+C_{\varepsilon} Q(x) u^{p-1}$. Let $\eta \in C_{0}^{1}\left(\Omega^{c}\right)$ with supp $\subset$ $(|x|>R)$, where $R>0$ is large and will be determined later. Taking $w=\eta^{2} u^{\beta}$, $\beta>0$, as a test function in (5.1) we obtain

$$
\beta \int_{\Omega^{c}} \eta^{2} u^{\beta-1}|\nabla u|^{2} d x+2 \int_{\Omega^{c}} \eta \nabla \eta \nabla u u^{\beta} d x \leq \int_{\Omega^{c}} d(x) \eta^{2} u^{\beta+1} d x .
$$

We now use the inequality

$$
\left|2 \int_{\Omega^{c}} \eta \nabla \eta \nabla u u^{\beta} d x\right| \leq \frac{\beta}{2} \int_{\Omega^{c}} \eta^{2}|\nabla u|^{2} u^{\beta-1} d x+\frac{2}{\beta} \int_{\Omega^{c}}|\nabla \eta|^{2} u^{\beta+1} d x,
$$


which inserted into (5.2) gives

$$
\frac{\beta}{2} \int_{\Omega^{c}} \eta^{2} u^{\beta-1}|\nabla u|^{2} d x \leq \int_{\Omega^{c}}\left(d(x) \eta^{2}+\frac{2}{\beta}|\nabla \eta|^{2}\right) u^{\beta+1} d x .
$$

We set $w=u^{(\beta+1) / 2}$ in (5.3) and we obtain

$$
\int_{\Omega^{c}} \eta^{2}|\nabla w|^{2} d x \leq \frac{(\beta+1)^{2}}{2 \beta} \int_{\Omega^{c}}\left(d(x) \eta^{2}+\frac{2}{\beta}|\nabla \eta|^{2}\right) w^{2} d x .
$$

We now estimate $\int_{\Omega^{c}} d(\eta w)^{2} d x$

$$
\begin{aligned}
\int_{\Omega^{c}} d(\eta w)^{2} d x= & \int_{\Omega^{c}} Q(p+\varepsilon) u_{\mu}^{p-1}(\eta w)^{2} d x+C_{\varepsilon} \int_{\Omega^{c}} Q u^{p-1}(\eta w)^{2} d x \\
\leq & (p+\varepsilon)\left\|u_{\mu}\right\|_{\infty}^{p-1}\left(\int_{\operatorname{supp} \eta} Q^{N / 2} d x\right)^{2 / N}\|\eta w\|_{2^{*}}^{2} \\
& +C_{\varepsilon} Q_{M, R}\left(\int_{\Omega^{c}} u^{2^{*}} d x\right)^{2 / N}\|\eta w\|_{2^{*}}^{2},
\end{aligned}
$$

where $Q_{M, R}=\sup _{|x|>R} Q(x)$. Setting

$$
M(R)=(p+\varepsilon)\left\|u_{\mu}\right\|_{\infty}^{p-1}\left(\int_{\operatorname{supp} \eta} Q^{N / 2} d x\right)^{2 / N}+C_{\varepsilon} Q_{M, R}\left(\int_{\Omega^{c}} u^{2^{*}} d x\right)^{2 / N}
$$

we rewrite the above inequality as

$$
\int_{\Omega^{c}} d(\eta w)^{2} d x \leq M(R)\|\eta w\|_{2^{*}}^{2}
$$

Also, we have

$$
\begin{aligned}
\left(\int_{\Omega^{c}}(\eta w)^{2^{*}} d x\right)^{(N-2) / N} & \leq S^{-1} \int_{\Omega^{c}}|\nabla(\eta w)|^{2} d x \\
& =S^{-1} \int_{\Omega^{c}}\left(\eta^{2}|\nabla w|^{2}+w^{2}|\nabla \eta|^{2}+2 \eta w \nabla \eta \nabla w\right) d x \\
& \leq 2 S^{-1} \int_{\Omega^{c}}\left(\eta^{2}|\nabla w|^{2}+w^{2}|\nabla \eta|^{2}\right) d x
\end{aligned}
$$

Inserting (5.5) into (5.4) we obtain

$$
\int_{\Omega^{c}} \eta^{2}|\nabla w|^{2} d x \leq \frac{(\beta+1)^{2}}{2 \beta} M(R)\|(\eta w)\|_{2^{*}}^{2}+\frac{(\beta+1)^{2}}{\beta^{2}} \int_{\Omega^{c}}|\nabla \eta|^{2} w^{2} d x .
$$

Combining the last inequality with (5.6) we get

$$
\begin{aligned}
\left(1-S^{-1} \frac{(\beta+1)^{2}}{\beta} M(R)\right)\left(\int_{\Omega^{c}}(\eta w)^{2^{*}} d x\right)^{(N-2) / N} & \\
& \leq 2 S^{-1}\left(1+\frac{(\beta+1)^{2}}{\beta^{2}}\right) \int_{\Omega^{c}}|\nabla \eta|^{2} w^{2} d x .
\end{aligned}
$$


We choose $R>0$ so that

$$
1-S^{-1} \frac{(\beta+1)^{2}}{\beta} M(R)=\frac{1}{2} .
$$

Thus

$$
\left(\int_{\Omega^{c}}(\eta w)^{2^{*}} d x\right)^{(N-2) / N} \leq A \int_{\Omega^{c}}|\nabla \eta|^{2} w^{2} d x,
$$

with $A=4 S^{-1}\left(1+(\beta-1)^{2} / \beta^{2}\right)$. We now make the following choice of $\eta$ : $\eta(x)=1$ in $B\left(x_{\circ}, r_{1}\right), \eta(x)=0$ in $\Omega^{c}-B\left(x_{\circ}, r_{2}\right),|\nabla \eta(x)| \leq 2 /\left(r_{2}-r_{1}\right)$ in $\Omega^{c}$, $1 \leq r_{1}<r_{2}<3$. It is assumed that $B\left(x_{\circ}, 3\right) \subset(|x|>R)$. Then (5.7) takes form

$$
\left(\int_{B\left(x_{\circ}, r_{1}\right)} w^{2^{*}} d x\right)^{(N-2) / 2 N} \leq \frac{A_{1}}{r_{2}-r_{1}}\left(\int_{B\left(x_{\circ}, r_{2}\right)} w^{2} d x\right)^{1 / 2},
$$

with $A_{1}=2 \sqrt{A}$. We set $\gamma=\beta+1, \chi=N /(N-2)$. Then we get from (5.8)

$$
\left(\int_{B\left(x_{\circ}, r_{1}\right)} u^{\gamma \chi} d x\right)^{1 /(\gamma \chi)} \leq\left(\frac{A_{1}}{r_{2}-r_{1}}\right)^{2 / \gamma}\left(\int_{B\left(x_{\circ}, r_{2}\right)} u^{\gamma} d x\right)^{1 / \gamma} .
$$

To iterate this inequality we take $s_{m}=1+2^{-m}, m=0,1, \ldots$ By a simple induction argument we get

$$
\begin{aligned}
& \left(\int_{B\left(x_{\circ}, s_{m}\right)} u^{\chi^{m} \gamma} d x\right)^{1 /\left(\gamma \chi^{m}\right)} \\
& \quad \leq A_{1}^{(2 / \gamma) \sum_{j=0}^{m-1}\left(1 / \chi^{j}\right)} 2^{(2 / \gamma) \sum_{j=0}^{m}(j+1) / \chi^{j}}\left(\int_{B\left(x_{\circ}, s_{0}\right.} u^{\gamma} d x\right)^{1 / \gamma}
\end{aligned}
$$

for each $m>1$. This inequality implies

$$
\begin{aligned}
& \left(\int_{B\left(x_{\circ}, 1\right)} u^{\chi^{m} \gamma} d x\right)^{1 /\left(\gamma \chi^{m}\right)} \\
& \quad \leq A_{1}^{(2 / \gamma) \sum_{j=0}^{m-1}\left(1 / \chi^{j}\right)} 2^{(2 / \gamma) \sum_{j=0}^{m}(j+1) / \chi^{j}}\left(\int_{B\left(x_{\circ}, 2\right)} u^{\gamma} d x\right)^{1 / \gamma} .
\end{aligned}
$$

We now choose $\gamma=\beta+1=2^{*}$. Letting $m \rightarrow \infty$ the result follows.

It follows from Proposition 5.1 that $\lim _{|x| \rightarrow \infty} u(x)=0$. By the maximum principle, since

$$
Q(x)\left(u+u_{\mu}\right)^{p}-Q(x) u_{\mu}^{p}>0
$$

we get $u(x) \geq C_{1}|x|^{2-N}$ for some constant $C_{1}>0$ and large $|x|$.

If $(\mathrm{H})$ holds, then assumptions of Proposition 5.1 are satisfied.

TheOREM 5.2. Suppose $(\mathrm{H})$ holds. Then problem $\left(1_{\mu}\right)$ has at least two solutions. 


\section{REFERENCES}

[1] S. Agmon, A. Douglis and L. Nirenberg, Estimates near the boundary for solutions of elliptic partial differential equations satisfying general boundary conditions I, Comm. Pure Appl. Math. XII, (959), 623-727.

[2] Adimurthi And G. Mancini, Geometry and topology of the boundary in the critical Neumann problem, J. Reine Angew. Math. 456 (1994), 1-18.

[3] H. BRÉZIS AND E. LIEB, A relation between point convergence of functions and convergence of functionals, Proc. Amer. Math. Soc. 88 (1983), 486-490.

[4] J. Chabrowski AND B. RuF, On the critical Neumann problem with weight in exterior domains, Nonlinear Anal. 54 (2003), 143-163.

[5] J. Chabrowski and M. Willem, Least energy solutions of a critical Neumann problem with a weight, Calc. Var. 15 (2002), 421-431.

[6] YinBin DENG AND Yi Li, On the existence of multiple positive solutions for a semilinear problem in exterior domains, J. Differential Equations 181 (2002), 197-229.

[7] R. DAutray And J. L. Lions, Mathematical analysis and numerical methods for science and technology, vol. 2, Springer, 1988.

[8] D. Gilbarg And N. S. Trudinger, Elliptic partial differential equations of second order, second ed., Springer, 1983.

[9] X.B. PAN AND X. WANG, Semilinear Neumann problem in exterior domains, Nonlinear Anal. 31 (1998), 791-821.

[10] P. L. Lions,, The concentration compactness principle in the calculus of variations. The limit case, Rev. Math. Iberoamericana 1 (1985), no. 1, 145-201; no. 2, 45-120.

[11] X. J. WANG, Neumann problems of semilinear elliptic equations involving critical Sobolev exponents, J. Differential Equations 93 (1991), 283-310.

JAN ChabRowski

Department of Mathematics,

University of Queensland

St. Lucia 4072, Qld, AUSTRALIA

E-mail address: jhc@maths.uq.edu.au

MiChel Willem

Institut de Mathématique Pure et Appliquée

Université Catholique de Louvain

1348 Louvain-la-Neuve, BELGIUM

E-mail address: willem@mth.ucl.ac.be

TMNA : Volume $26-2005-\mathrm{N}^{\circ} 1$ 